\title{
THE EXISTENCE OF HARMONIC VIBRATIONS
}

\author{
B. VISWANATHAM
}

1. In what follows we shall consider a system of nonlinear differential equations given by

$$
\dot{x}=X(x, t),
$$

where $x$ and $X$ are column vectors of $m$ components each. $X$ is a sufficiently smooth vector function, continuous in the region $0 \leqq t<\infty$; $-\infty<x<+\infty$ so that the usual theorems of existence and uniqueness hold good. We shall further suppose that $X$ is periodic in $t$ with period unity. It is known that such systems may admit solutions of various periods, but solutions whose period is the same as that of the system are of particular importance for application to physical problems. Such solutions are called harmonic vibrations. For $m=2$, Norman Levinson [1] proved the existence of harmonic vibrations for dissipative systems. J. L. Massera [2] in a more recent paper considered the existence of harmonic vibrations with less stringent conditions for $m=1$ and $m=2$, and says that for $m>2$ the problem awaits a solution. It is proposed to consider this problem here.

2. Definitions.

(i) If $P$ and $Q$ are two points in $m$-dimensional Euclidean space, we shall say $P \geqq Q$ whenever the same relation holds between their respective components.

(ii) We shall say that a vector is monotonic if every one of its components is monotonic.

THEOREM. If at least one solution of (1.1) is bounded and monotonic (increasing or decreasing) there is a harmonic vibration.

The mere boundedness of a solution is not enough to ensure the existence of a harmonic vibration as was shown by Massera [2] by a counter example. Some sort of an additional condition is necessary.

For the proof of this theorem we shall use the following well known lemma of Zorn [3].

Lemma. Let $E$ be an inductively ordered set, and $f$ a transformation from $E$ into $E$ such that for any $x$ belonging to $E$ we have $f(x) \geqq x$. Then there is at least one point belonging to $E$ such that $f(x)=x$.

Proof of the theorem. Let $P_{0}$ be a point in the $m$-dimensional

Received by the editors May 20, 1952 and, in revised form, August 18, 1952. 
Euclidean space. Through $P_{0}$ there is a solution of (1.1) passing for $t=t_{0}$. Denote this solution by $x_{i}(t)(i=1,2, \cdots, m)$. Take the point $x_{i}\left(t_{0}+1\right)(i=1,2, \cdots, m)$ on this solution curve and denote it by $P_{1}$. We shall define a transformation $T$ as one which takes any point $P_{0}$ into $P_{1}$ obtained by the above process. Since $X$ is periodic in $t$ with period unity, any solution passing through a fixed point under the above transformation is obviously a harmonic vibration. Hence it is enough to prove the existence of a fixed point under the above transformation.

Now let $P_{0}$ be the initial point of the bounded and monotonic solution $x_{i}(t)(i=1,2, \cdots, m)$. Denote the point $x_{i}(n)(i=1,2, \cdots, m)$ by $P_{n}$. It is clear that each point $P_{n}$ is the image of the preceding point under the transformation $T$. Since these points form a bounded, monotonic and denumerably infinite set, Zorn's lemma is applicable and we have at least one point $P$ such that $P=T(P)$. It is clear that the solution of the system through $P$ is a harmonic vibration.

Note. It should be noted that the monotonic character of the successive images alone is needed for the proof of the theorem. The solution itself need not be monotonic. It is enough if the points $P_{n}$ $(n=1,2, \cdots)$ on the solution curve be monotonic. Theorem 1 of Massera's paper seems to be a particular case of this theorem. For $m=1$ the monotonic character of the successive images is implied by the uniqueness of the solution, which is not so for $m>1$.

\section{REFERENCES}

1. Norman Levinson, Transformation theory of non-linear differential equations of second order, Ann. of Math. (2) vol. 45 (1944) pp. 723-737.

2. J. L. Massera, The existence of periodic solutions of systems of differential equations, Duke Math. J. vol. 17 (1950) pp. 457-475.

3. N. Bourbaki, Topologie generale, p. 37.

OsMaNia UNIVERSITY 